04

\title{
Поглощательная способность слоя нанокомпозита со сферическими металлическими включениями
}

\author{
() С.В. Елисеева, Д.И. Семенцов \\ Ульяновский государственный университет, \\ 432970 Ульяновск, Россия \\ e-mail: eliseeva-sv@yandex.ru
}

Поступила в редакцию 02.01.2018 г.

В окончательной редакции 10.02.2018 г.

\begin{abstract}
Исследована зависимость поглощательной способности слоя нанокомпозита (диэлектрическая матрица со сферическими металлическими включениями) от частоты, объемной доли и размера включений, толщины слоя и угла падения волны на него. Показано, что особенности оптических характеристик связаны с плазмонным резонансом в наночастицах и резонансной частотной зависимостью диэлектрической проницаемости композитной среды. Выявлены значения параметров пленки и частотные интервалы, где падающее излучение практически полностью поглощается.
\end{abstract}

DOI: 10.21883/OS.2018.06.46088.1-18

\section{Введение}

Управление поглощаемой энергией падающего на структуру излучения является одной из ключевых проблем в целом ряде прикладных задач нанофотоники. Поэтому чрезвычайно важным является поиск материалов и оптимизация параметров поглотителей энергии заданного частотного диапазона [1-6]. В общем случае поглощаемая конкретным образцом энергия зависит как от его параметров, так и от параметров излучения (длины волны, угла падения). Типичной является ситуация, когда для поглощения падающего излучения поглотитель выбирается в виде плоского слоя поглощающего вещества. Однако расположенный в вакууме однородный тонкий слой не может поглотить более 50\% падающего излучения [7].

Для увеличения поглощающей способности требуется более сложная структура слоя-поглотителя. В частности, поглотители делают неоднородными по составу с использованием различных метаматериалов [8,9]. В последнее время внимание исследователей привлекают металлодиэлектрические нанокомпозитные (НК) среды, представляющие собой диэлектрическую матрицу с равномерно распределенными по ее объему металлическими наночастицами [10-23]. Эффективные материальные параметры таких НК сред могут значительно отличаться от соответствующих характеристик как включений, так и матрицы композита, принимая значения, не присущие исходным материалам.

Необычные оптические характеристики НК среды формируются благодаря поверхностно-плазмонному резонансу металлических наночастиц, положение и форма линии которого зависит от диэлектрической проницаемости (ДП) исходных материалов, концентрации и размера наночастиц. Так, наличие серебряных наночастиц в диэлектрической матрице (стекле) приводит к появлению в оптических спектрах пропускания НК сред селективных полос поглощения в видимой области спектра в диапазоне длин волн 400-450 nm [24-26].

Варьирование параметрами нановключений позволяет в достаточно широких пределах изменять действительную и мнимую части эффективной ДП, что позволяет управлять оптическими характеристиками НК среды, в том числе и поглощением [14-16]. В настоящей работе исследуются особенности поглощения пленки нанокомпозита с однородно распределенными металлическими включениями сферической формы. Для записи ДП металлических включений используется приближение Друде с учетом поглощения и размера наночастиц [17-19], а для НК среды используется приближение эффективной среды [20-23].

\section{Материальные параметры}

Рассмотрим тонкую пленку, выполненную из композитного материала „ $\mathrm{SiO}_{2} /$ серебро“, который представляет собой распределенные в диоксиде кремния с ДП $\varepsilon_{1 m}=2.15$ наноразмерные сферические частицы серебра с объемной долей $\eta$ и радиусом $a$. Параметры серебра: $v_{F}=1.4 \cdot 10^{6} \mathrm{~m} / \mathrm{s}, \varepsilon_{0}=4.96, \omega_{p}=1.367 \cdot 10^{16} \mathrm{~s}^{-1}$, $\gamma_{0}=3.04 \cdot 10^{13} \mathrm{~s}^{-1}$ [21-23]. При исследовании оптических свойств НК сред необходимо, чтобы толщина пленки $h$ в несколько раз превышала усредненное расстояние между металлическими частицами $d=a(4 \pi / 3 \eta)^{1 / 3}$ (чтобы рассеяние волны происходило на нескольких слоях нановключений). Так, для используемых далее значений $\eta=0.1$ и $a=10 \mathrm{~nm}$ расстояние между центрами наночастиц $d=35 \mathrm{~nm}$.

Согласно модели эффективной среды Максвелла-Гарнетта, ДП пленки, состоящей из одинаковых металлических наночастиц сферической формы, однородно распределенных в диэлектрической матрице, может быть 
представлена в виде [20,21]

$$
\varepsilon_{\mathrm{ef}}=\varepsilon_{m}\left(1+\frac{\eta\left(\varepsilon_{p}-\varepsilon_{m}\right)}{\varepsilon_{m}+(1-\eta)\left(\varepsilon_{p}-\varepsilon_{m}\right) g}\right),
$$

где $\varepsilon_{m}$ и $\varepsilon_{p}-$ ДП матрицы и включений соответственно, $g$ - геометрический фактор, который для сферических частиц равен $1 / 3$. Диэлектрическая проницаемость диэлектрика, используемого в качестве матрицы композита, будем считать постоянной и действительной величиной. Для проницаемости металлических наночастиц используем выражение

$$
\varepsilon_{p}(\omega)=\varepsilon_{0}-\frac{\omega_{p}^{2}}{\omega^{2}+i \omega \gamma(a)},
$$

где $\omega_{p}-$ плазменная частота свободного электронного газа в неограниченном объеме, $\varepsilon_{0}-$ вклад ионной решетки в ДП металла, $\gamma$ - скорость релаксации, которая совпадает с шириной линии плазмонного резонанса.

Известно, что ширина линии плазмонного резонанса для сферических наночастиц обратно пропорциональна их радиусу $a$. Когда средняя длина пробега электронов сравнивается или начинает превосходить размер наночастиц, в релаксацию существенный вклад вносят процессы рассеяния электронов на поверхности наночастицы, поэтому релаксационный параметр в (2) начинает зависеть от радиуса наночастицы [17]:

$$
\gamma(a)=\gamma_{0}+D \frac{v_{F}}{a},
$$

где $\gamma_{0}$ - константа затухания для неограниченного объема металла, $v_{F}-$ скорость электронов на поверхности Ферми. Коэффициент $D$ определяется деталями процесса рассеяния электронов на поверхности наночастиц и имеет значение, близкое к единице. Поскольку коэффициент $D$ не имеет однозначного теоретического выражения, его часто полагают равным единице.

Учет релаксации в выражении (2) приводит к комплексности компонент тензора эффективной проницаемости нанокомпозита $\varepsilon_{\mathrm{ef}}=\varepsilon_{\mathrm{ef}}^{\prime}+i \varepsilon_{\mathrm{ef}}^{\prime \prime}$, где действительная и мнимая части в соответствии с (1) определяются следуюшими выражениями:

$$
\begin{gathered}
\varepsilon_{\mathrm{ef}}^{\prime}=\varepsilon_{m}+\frac{\eta}{G}\left\{\left(\varepsilon_{p}^{\prime}-\varepsilon_{m}\right)\left(1+g(1-\eta) \frac{\varepsilon_{p}^{\prime}-\varepsilon_{m}}{\varepsilon_{m}}\right)\right. \\
\left.+g(1-\eta) \frac{\left(\varepsilon_{p}^{\prime \prime}\right)^{2}}{\varepsilon_{m}}\right\}, \quad \varepsilon_{\mathrm{ef}}^{\prime \prime}=\frac{\eta}{G} \varepsilon_{p}^{\prime \prime}, \\
G=\left(1+g(1-\eta) \frac{\varepsilon_{p}^{\prime}-\varepsilon_{m}}{\varepsilon_{m}}\right)^{2}+\left(g(1-\eta) \frac{\varepsilon_{p}^{\prime \prime}}{\varepsilon_{m}}\right)^{2} .
\end{gathered}
$$

Здесь введены обозначения

$\varepsilon_{p}^{\prime}=\varepsilon_{0}-\frac{\omega_{p}^{2}}{\omega^{2}+\left(\gamma_{0}+v_{F} / a\right)^{2}}, \quad \varepsilon_{p}^{\prime \prime}=\frac{\left(\gamma_{0}+v_{F} / a\right) \omega_{p}^{2}}{\omega\left(\omega^{2}+\left(\gamma_{0}+v_{F} / a\right)^{2}\right)}$.

На рис. 1 приведена частотная зависимость действительной и мнимой частей эффективной ДП нанокомпозита с параметрами: $a=10 \mathrm{~nm}$ и $\eta=0.01,0.02,0.05,0.1$
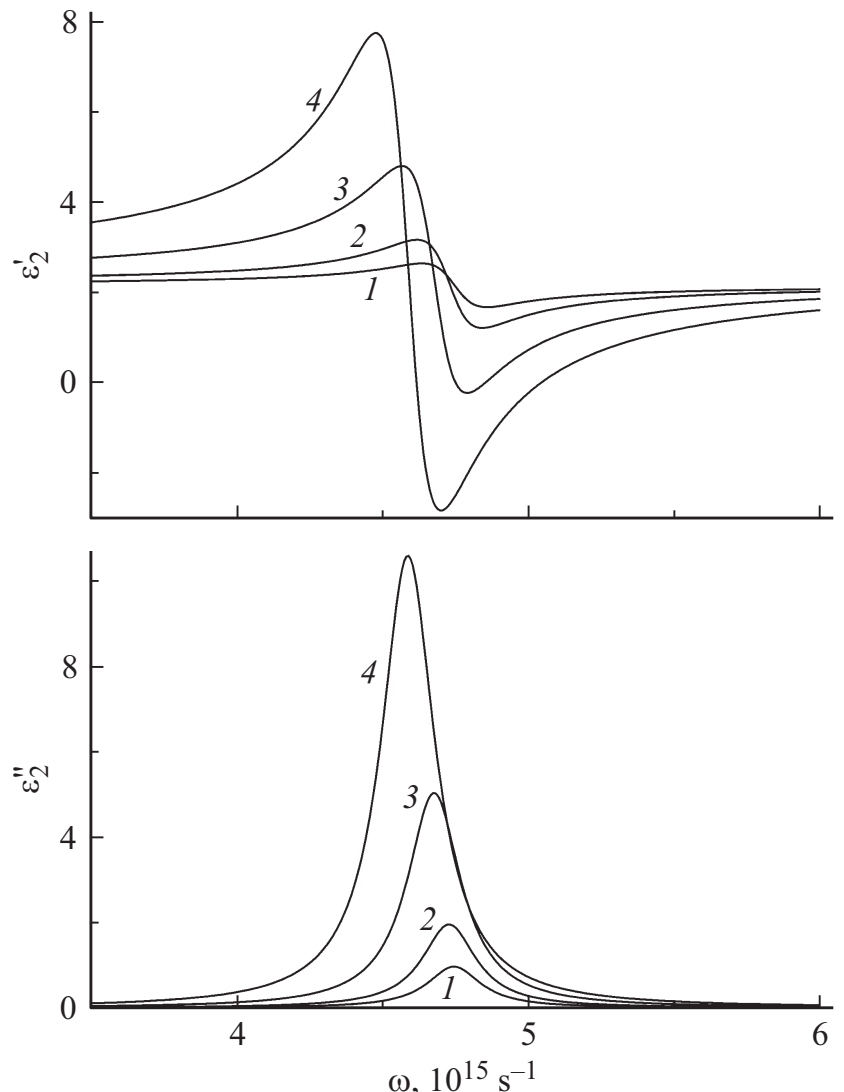

Рис. 1. Частотная зависимость действительной и мнимой частей эффективной диэлектрической проницаемости нанокомпозита, $a=10 \mathrm{~nm}, \eta=0.01$ (1), 0.02 (2), 0.05 (3), 0.1 (4).

(кривые 1-4 соответственно). Указанные зависимости имеют резонансный характер, связанный с плазмонным резонансом наночастиц. Резонансная частота нанокомпозита отвечает максимуму мнимой части эффективной ДП:

$$
\omega_{\mathrm{res}} \approx \sqrt{\frac{(1-\eta) g \omega_{p}^{2}}{\varepsilon_{m}+(1-\eta) g\left(\varepsilon_{0}-\varepsilon_{m}\right)}-\left(\gamma_{0}+\frac{v_{F}}{a}\right)^{2}},
$$

и для выбранных значений параметров $\omega_{\text {res }}$ лежит в оптическом диапазоне. С увеличением доли включений $\eta$ наблюдается сдвиг резонансной линии в область более низких частот и рост максимальных значений как действительной, так и мнимой частей эффективной ДП. При этом появляется интервал частот $\omega_{1}<\omega<\omega_{2}$, где $\varepsilon_{\text {ef }}^{\prime}$ становится отрицательной. В соответствии с выражениями (4) для указанных частот получаем

$\omega_{j}=\omega_{p}\left[\varepsilon_{0}-\varepsilon_{m}\left(1-\frac{1}{g(1-\eta)+(j-1) \eta}\right)\right]^{-1 / 2}, \quad j=1,2$.

На рис. 2 приведена зависимость резонансной частоты от размера наночастиц серебра, полученная для значений $\eta=0.01,0.02,0.05,0.1$ (кривые $1-4$ соответственно). Штриховая линия иллюстрирует зависимость 


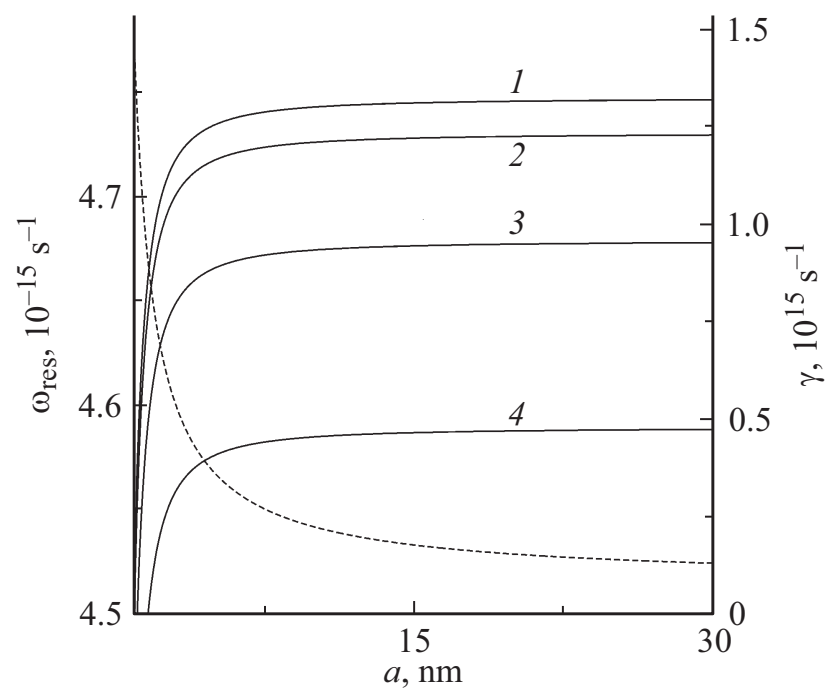

Рис. 2. Зависимость резонансной частоты (сплошные кривые) и параметра релаксации (пунктирная кривая) от размера включений для различных значений доли включений $\eta=0.01(1)$, 0.02 (2), 0.05 (3), 0.1 (4).

параметра релаксации $\gamma$ от размера наночастиц. Видно, что с увеличением размера наночастиц в области значений $a<4 \mathrm{~nm}$ наблюдается резкий рост резонансной частоты, тогда как при $a>10 \mathrm{~nm} \omega_{\text {res }}$ практически постоянна. С увеличением доли включений резонансная частота уменьшается в соответствии с выражением (5). Параметр релаксации растет с уменьшением размера наночастиц: в области $a<4 \mathrm{~nm}$ имеет место его быстрый рост, а при $a>20 \mathrm{~nm} \gamma$ является уже слабо меняющейся величиной. В таблице для различных долей включений и размеров наночастиц серебра $a=10 \mathrm{~nm}$ даны значения резонансной частоты и частот, на которых действительная часть ДП достигает своего максимального и минимального значений.
Значения частот для характерных значений ДП нанокомпозита

\begin{tabular}{c|c|c|c}
\hline$\eta$ & $\omega_{\varepsilon_{\max }} \cdot 10^{-15}, \mathrm{~s}^{-1}$ & $\omega_{\text {res }} \cdot 10^{-15}, \mathrm{~s}^{-1}$ & $\omega_{\varepsilon_{\min }} \cdot 10^{-15}, \mathrm{~s}^{-1}$ \\
\hline 0.01 & 4.65 & 4.76 & 4.86 \\
0.02 & 4.62 & 4.74 & 4.85 \\
0.05 & 4.57 & 4.68 & 4.80 \\
0.1 & 4.49 & 4.598 & 4.71
\end{tabular}

\section{Оптические характеристики}

Пусть плоская линейно поляризованная монохроматическая волна с амплитудой $E_{0}$ падает под углом $\theta_{1}$ на тонкую пленку НК среды толщиной $h$ и ДП $\varepsilon_{2}$. Пленка разделяет две среды, непоглощающие в рассматриваемом диапазоне длин волн, с ДП $\varepsilon_{1}=\varepsilon_{3}$. Направим ось $Z$ вдоль нормали к границе раздела сред, плоскость $X Z$ является плоскостью падения.

Энергетические коэффициенты отражения и пропускания параллельной и ортогональной плоскости падения поляризаций $(\alpha=p, s)$ для рассматриваемой структуры определяются общими выражениями [27]:

$$
R_{\alpha}=\left|r_{\alpha}\right|^{2}, \quad T_{\alpha}=\frac{\sqrt{\varepsilon_{1}} \cos \theta_{1}}{\sqrt{\varepsilon_{3}} \cos \theta_{3}}\left|t_{\alpha}\right|^{2},
$$

где $r_{\alpha}$ и $t_{\alpha}-$ амплитудные коэффициенты отражения и пропускания изотропной пленки, $\theta_{i}-$ угол падения в соответствующей среде. С учетом симметрии структуры $T_{\alpha}=\left|t_{\alpha}\right|^{2}$. Наличие поглощения в пленке нанокомпозита приводит к тому, что часть падающей на пленку энергии переходит в тепло. В этой связи интерес представляет коэффициент поглощения $A_{\alpha}=1-R_{\alpha}-T_{\alpha}$, определяющий долю поглощенной пленкой энергии. При нормальном падении излучения на пленку изотропного нанокомпозита выражения соответствующих коэффициентов одинаковы для обеих поляризаций.
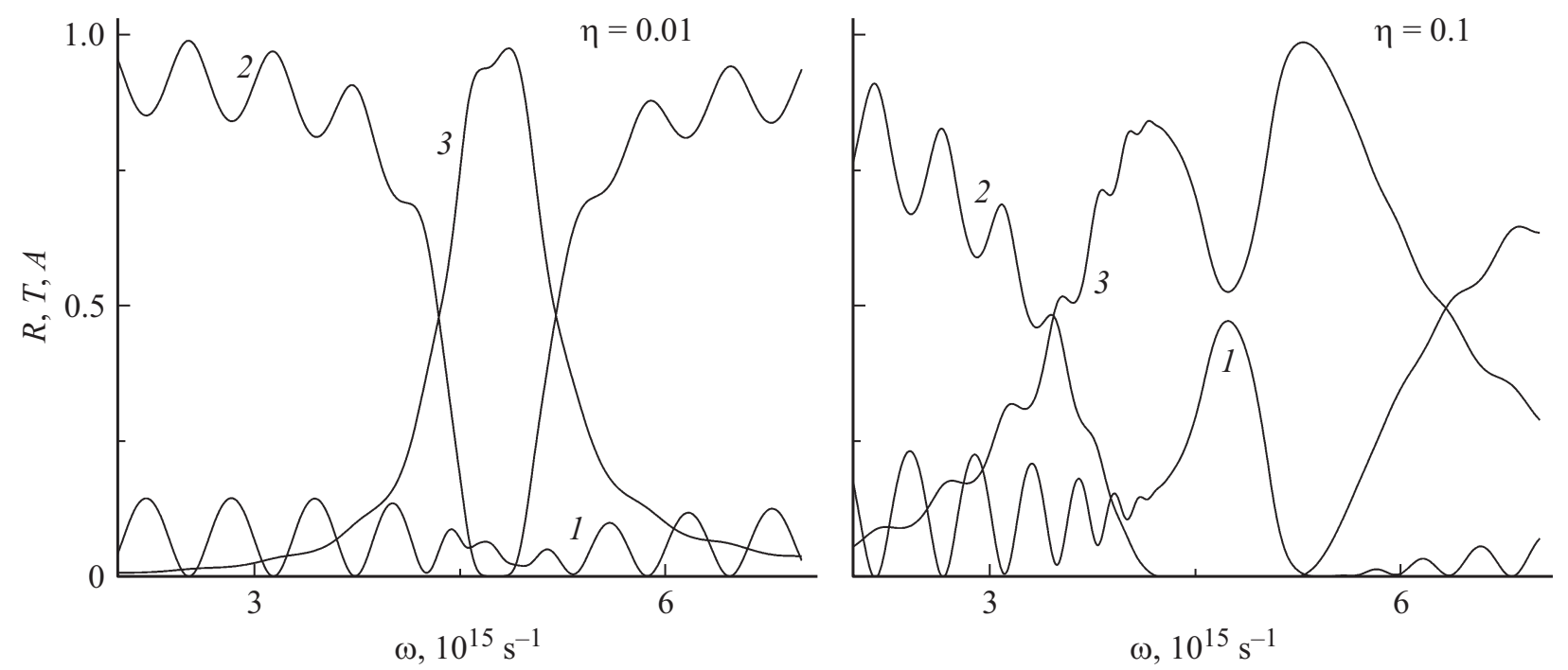

Рис. 3. Частотная зависимость коэффициентов отражения (1), пропускания (2) и поглощения (3), $a=10 \mathrm{~nm}, \eta=0.01,0.1$. 


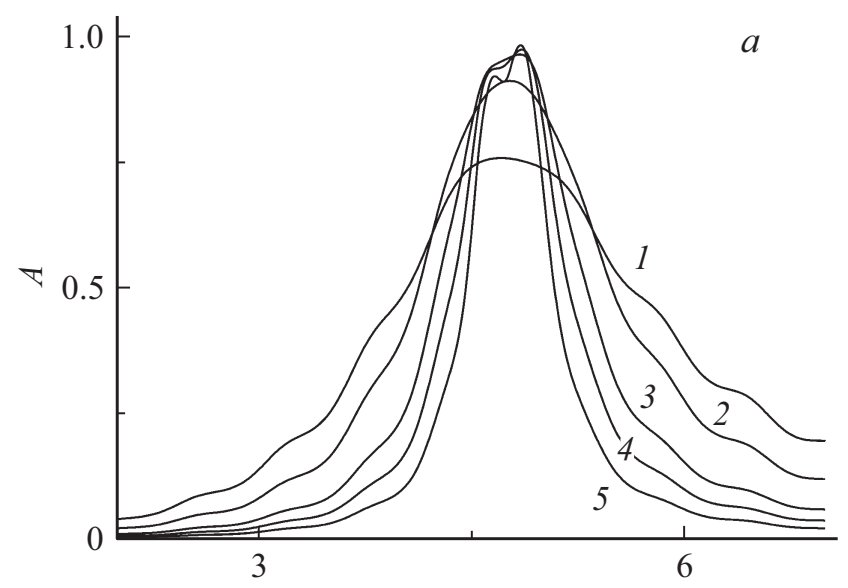

$\omega, 10^{15} \mathrm{~s}^{-1}$

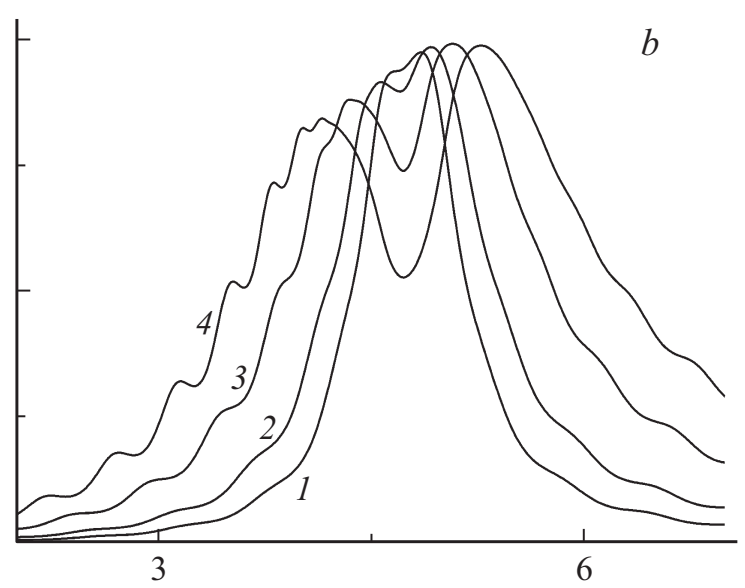

$\omega, 10^{15} \mathrm{~s}^{-1}$

Рис. 4. Частотная зависимость коэффициента поглощения для толщины пленки $h=1 \mu \mathrm{m}:(a) \eta=0.01, a=1$ (1), 2 (2), 5 (3), $10(4), 30 \mathrm{~nm}(5) ;(b) a=10 \mathrm{~nm}, \eta=0.01(1), 0.02(2), 0.05(3), 0.1$ (4).
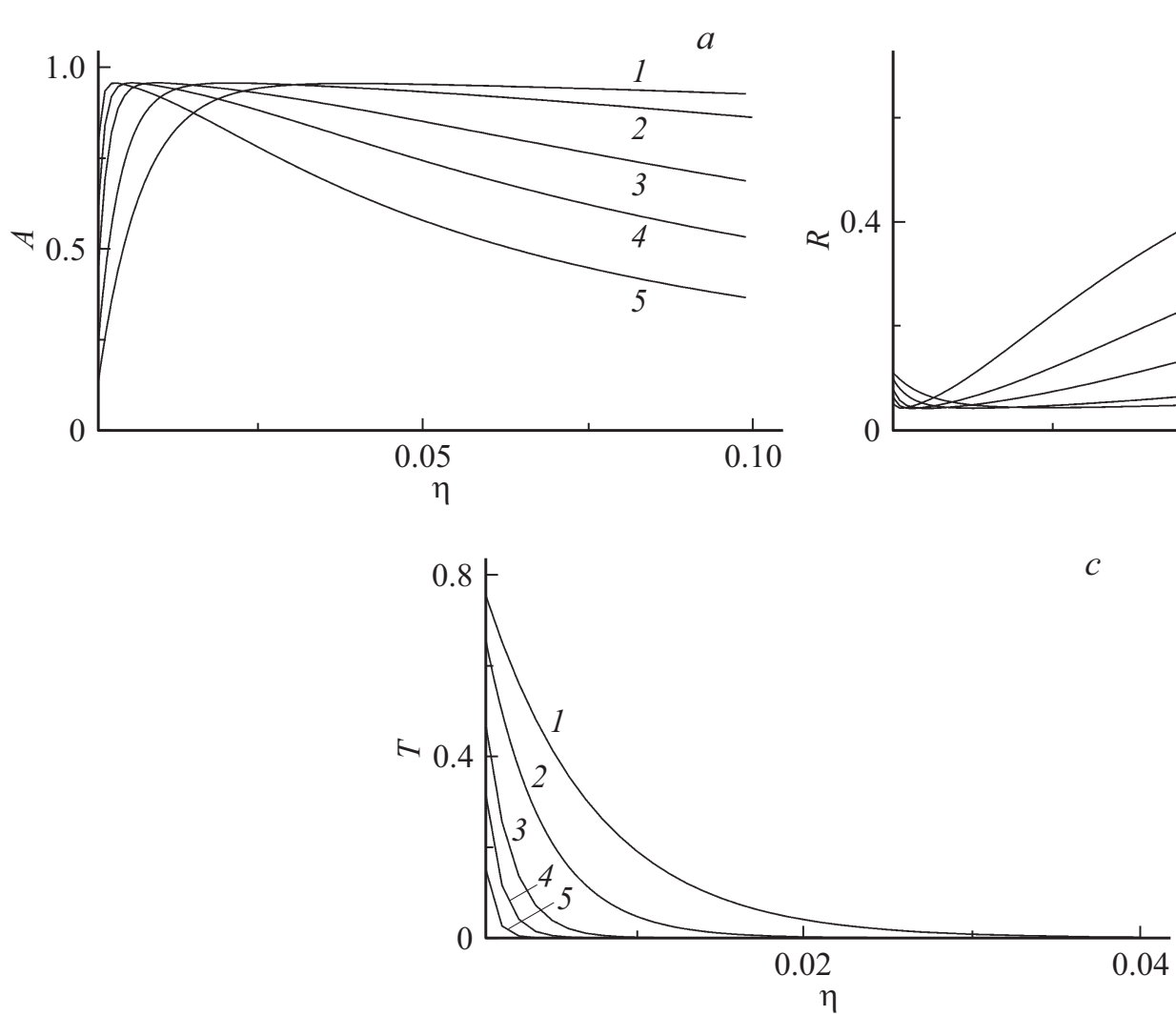

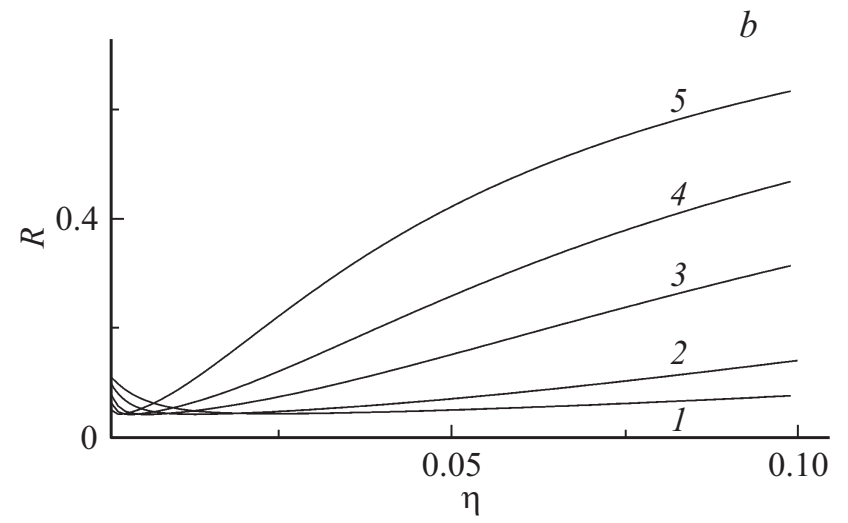

Рис. 5. Зависимость от доли включений энергетических коэффициентов поглощения, отражения и пропускания при толщине пленки $h=1 \mu \mathrm{m}$ на резонансной частоте $4.76 \cdot 10^{15} \mathrm{~s}^{-1}$ для размера включений $a=1(1), 2(2), 5(3), 10(4), 30 \mathrm{~nm}(5)$.

\section{Численный анализ}

Ниже представлены результаты численного анализа приведенных выше соотношений для энергетических коэффициентов пленки нанокомпозита с различными размерами включений и их объемной доли. На рис. 3 приведена частотная зависимость коэффициентов отражения, пропускания и поглощения (кривые 1-3) при нормаль- ном падении волны на пленку НК толщиной $h=1 \mu \mathrm{m}$, размером наночастиц $a=10 \mathrm{~nm}$ и объемной долей $\eta=0.01,0.1$. Видно, что при малой объемной доле в резонансной области поглощение максимально, $A=0.96$ (коэффициент поглощения близок к единице), отражение мало, $R=0.04$, а пропускание вообще отсутствует, $T=0$. Вне резонансной области наблюдаются осцилляции коэффициентов отражения и поглощения. С увели- 

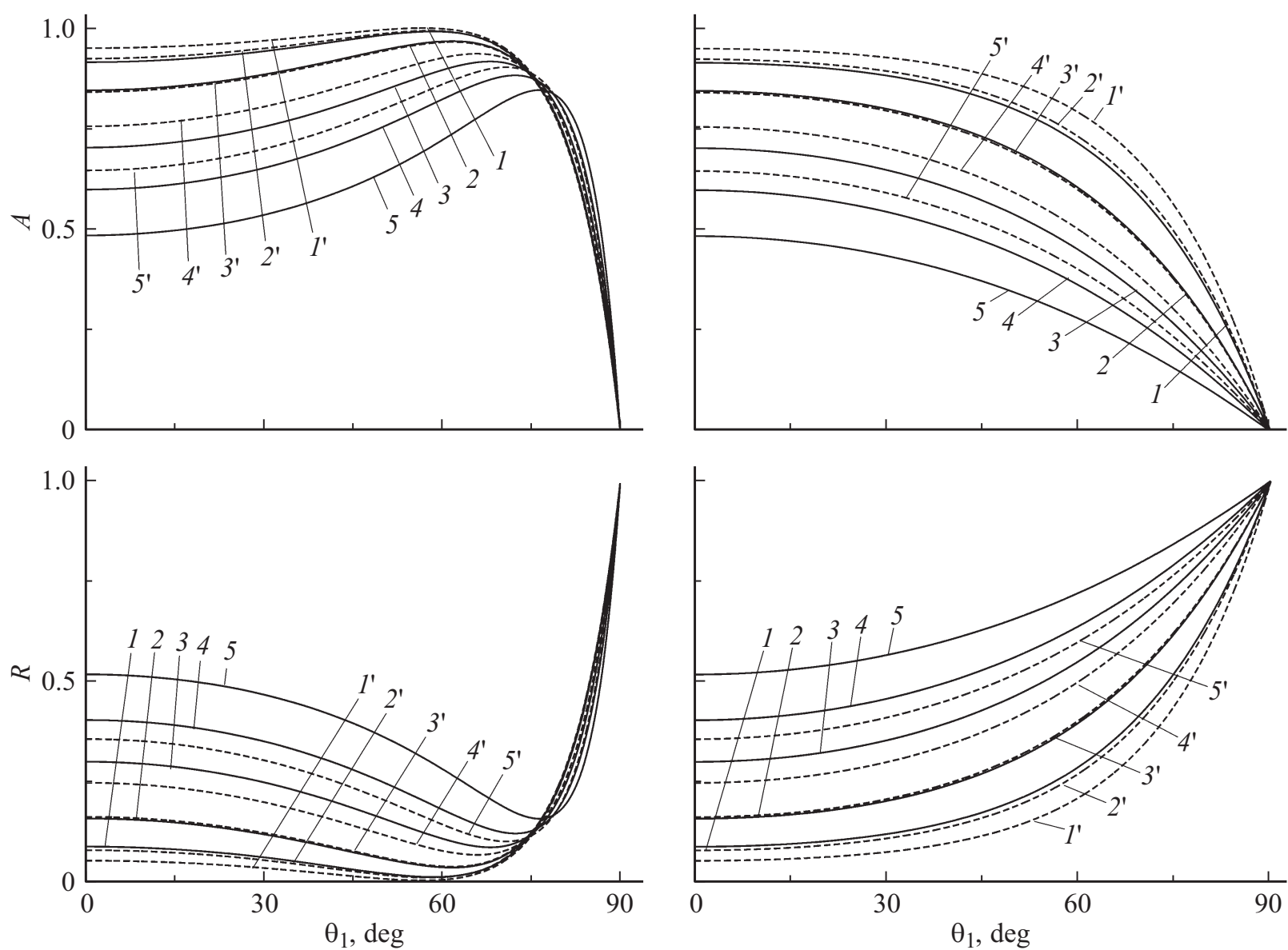

Рис. 6. Угловая зависимость коэффициента поглощения и отражения при $\eta=0.1$ (сплошные кривые $1-5$ ), 0.05 (пунктирные кривые $1^{\prime}-5^{\prime}$ ) (сплошные и пунктирные линии соответствуют размерам нановключений $\left.a=1,2,5,10,30 \mathrm{~nm}\right)$ на резонансной частоте $4.76 \cdot 10^{15} \mathrm{~s}^{-1}$, при толщине пленки $h=1 \mu \mathrm{m}$, для $p$ - (слева) и $s$-поляризаций (справа).

чением концентрации наночастиц в резонансной области наблюдается значительный рост отражения и уменьшение поглощения при полном отсутствии пропускания.

Области с коэффициентом поглощения, близким к единице, характерны для пленок НК сред независимо от размера включений и концентрации наночастиц. На рис. 4 представлена частотная зависимость коэффициента поглощения для пленки с $h=1 \mu \mathrm{m}$ при следующих значениях параметров: $\eta=0.01, a=1,2,5,10$, $30 \mathrm{~nm}$ ( $a$, кривые $1-5)$ и $a=10 \mathrm{~nm}, \eta=0.01,0.02$, $0.05,0.1$ ( $b$, кривые $1-4)$. При фиксированном значении параметра $\eta$ с увеличением размера наночастиц форма линии поглощения становится более узкой, а поглощение максимальным. При фиксированном размере наночастиц с увеличением доли включений на резонансной частоте поглощение уменьшается, а максимум поглощения смещается в область более высоких частот. Для значений $\eta=0.1$ и $a=10 \mathrm{~nm}$ имеется область почти полного поглощения. При уменьшении доли включений эта область становится меньше и смещается в сторону более низких частот.

На рис. 5 представлена зависимость коэффициентов поглощения, отражения и пропускания от объемной доли включений, полученная на резонансной частоте для размера включений $a=1,2,5,10,30 \mathrm{~nm}$ (кривые 1-5). Обращает внимание то, что на указанной частоте при концентрациях включений $\eta \geq 0.01$ и всех реалистичных размерах наночастиц $(a>3 \mathrm{~nm})$ коэффициент пропускания практически равен нулю, т.е. пленка НК среды толщиной $h=1 \mu \mathrm{m}$ при нормальном падении на нее излучения становится непрозрачной. При этом большая часть падающей на пленку энергии поглощается. Наибольшее поглощение достигается при малых концентрациях $\eta<0.01$, с ростом концентрации и размера наночастиц поглощение начинает плавно уменьшаться, а отражение растет. При больших концентрациях $\eta \geq 0.1$ практически полного поглощения можно достичь при малых размерах включений $a \leq 1 \mathrm{~nm}$.

На рис. 6 представлены угловые зависимости коэффициента поглощения и отражения для $p$ - и $s$-поляризаций волны, падающей на пленку толщиной $h=1 \mu \mathrm{m}$. Полученные зависимости отвечают резонансной частоте при $\eta=0.1, a=1,2,5,10,30 \mathrm{~nm}$ (сплошные кривые $1-5$ ) и $\eta=0.05, a=1,2,5,10,30 \mathrm{~nm}$ (пунктирные кривые $l^{\prime}-5$ '). Для $p$-поляризации при всех значениях параметра $a$ наблюдается максимум поглощения на 

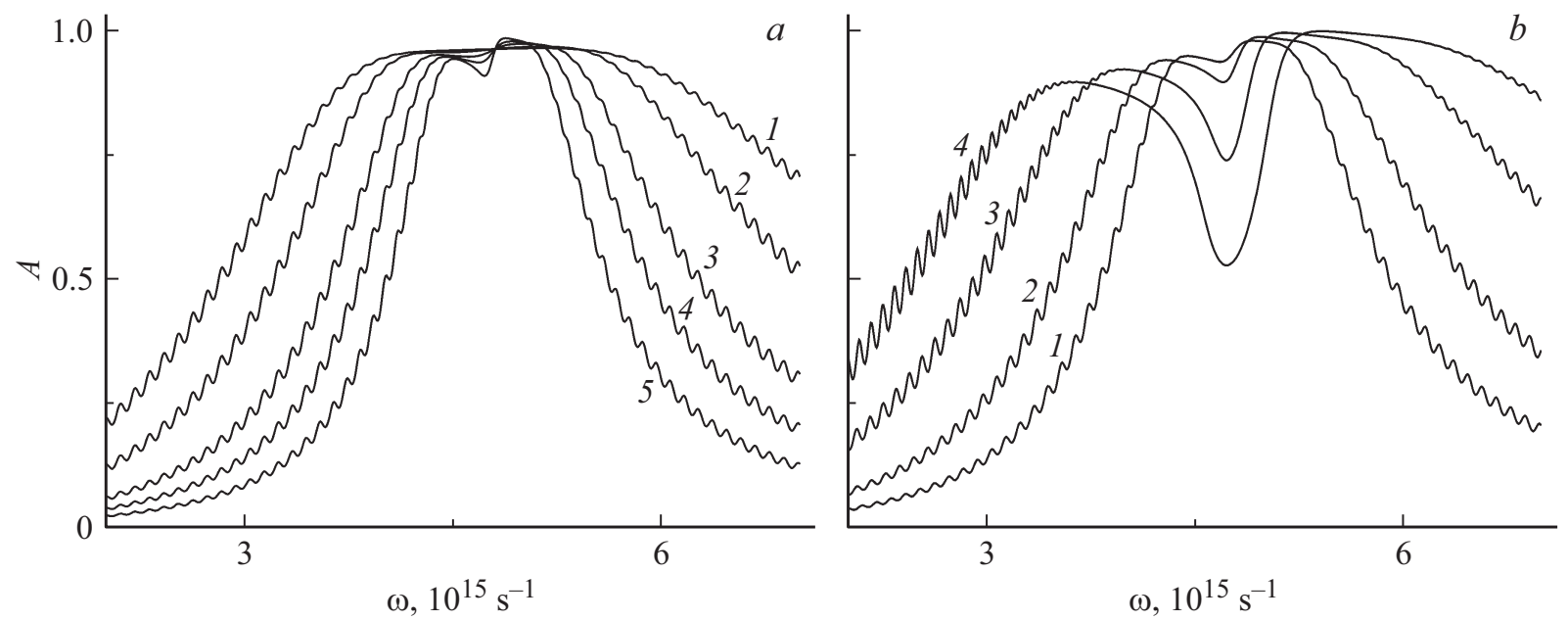

Рис. 7. Частотная зависимость коэффициента поглощения при толщине пленки $h=6 \mu \mathrm{m}:(a) \eta=0.01, a=1$ (1), 2 (2), 5 (3), 10 (4), $30 \mathrm{~nm}(5) ;(b) a=10 \mathrm{~nm}, \eta=0.01$ (1), 0.02 (2), 0.05 (3), 0.1 (4).

угле, при котором отражение становится минимальным или даже отсутствует (при $a=1 \mathrm{~nm}$ ). Этот угол можно считать углом Брюстера для поглощающей НК структуры. Для $s$-поляризации подобный угол отсутствует, и зависимости коэффициентов поглощения и отражения имеют монотонный характер. При этом для всех значений концентрации включений р-поляризованные волны имеют большее поглощение, чем волны s-поляризации. Отметим также, что пропускание для волн обеих поляризаций очень мало.

На рис. 7 представлена частотная зависимость коэффициента поглощения для пленки толщиной $h=6 \mu \mathrm{m}$, остальные параметры взяты теми же, что и на рис. 4. Из сравнения зависимостей, приведенных на обоих рисунках, следует, что с увеличением толщины пленки происходит уширение области повышенного поглощения (вблизи резонансной частоты) и увеличение частоты осцилляций коэффициента поглощения вне резонансной области. Наблюдается также общее увеличение поглощения на рассматриваемом интервале частот.

\section{Заключение}

Исследованы особенности оптических характеристик, в частности, поглощательной способности слоя композита с металлическими сферическими наночастицами (серебро), которые проявляются при изменении их размера и объемной доли, при нормальном и наклонном падении излучения на слой. Из полученных зависимостей следует, что в спектрах энергетических коэффициентов есть частоты, на которых поглощение максимально, а отражение и пропускание практически отсутствуют. Это позволяет использовать пленку нанокомпозита в качестве поглотителя излучения. При уменьшении размеров включений и постоянной объемной доле увеличивается частотная область, на которой поглощение максимально. Также в спектре имеется область частот, в которой пропускание отсутствует, что позволяет использовать тонкую пленку в качестве частотного фильтра. При увеличении толщины пленки частотная область сильного поглощения расширяется, при этом увеличиваются осцилляции коэффициента поглощения вне этой области. Наблюдаемые особенности оптических свойств пленки НК среды указывают на хорошие возможности практического применения их в фотонике.

Работа выполнена в рамках реализации ГК № 14.Z50.31.0015 и № 3.6825.2017/БЧ Министерства образования и науки РФ.

\section{Список литературы}

[1] Борен К., Хафмен Д. Поглощение и рассеяние света малыми частицами. Пер. с англ. М.: Мир, 1986. 664 с.

[2] Ganeev R.A., Ryasnyansky A.I., Stepanov A.L., Usmanov T. // Opt. Quant. Electron. 2004. V. 36. N 10. P. 949-960.

[3] Kachan S., Stenzel O., Ponyavina A. // Appl. Phys. B. 2006. V. 84. P. 281-287.

[4] Kravets V.G., Schedin F., Grigorenko A.N. // Phys. Rev. B. 2008. V. 78. P. 205405-20541.

[5] Климов В.В. Наноплазмоника. М.: ФИЗМАТЛИТ, 2009. $480 \mathrm{c}$.

[6] Tretyakov S.A. // Plasmonics. 2013. V. 9. P. 935.

[7] Radi Y., Simovski C.R., Tretyakov S.A. // Phys. Rev. Appl. 2015. V. 3. P. 037001.

[8] Cai W., Shalaev V. Optical Metamaterials: Fundamentals and Applications. Springer, 2010. 200 p.

[9] Engheta N., Ziolkowski R.W. Metamaterials: Physics and Engineering Explorations. Wiley-IEEE Press, 2006. 352 p.

[10] Baffou G., Quidant R. // Las. Photon. Rev. 2013. V. 7. P. 171187.

[11] Sentenac A., Chaumet P.C., Leuchs G. // Opt. Lett. 2013. V. 38. P. $818-820$.

[12] Grigoriev V., Bonod N., Wenger J., Stout B. // ACS Photonics. 2015. V. 2. P. 263. 
[13] Miller O.D., Hsu C.W., Reid M., Qiu W., DeClay B., Joannopoulos J.D., Soljačić M., Johnson S. // Phys. Rev. Lett. 2014. V. 112. P. 123903.

[14] Ряснянский A.И., Palpant B., Debrus S. и др. // ФТТ. 2009. T. 51. C. 52.

[15] Ветров С.Я., Авдеева А.Ю., Тимофеев И.В. // ЖЭТФ. 2011. T. 140. C. 871.

[16] Моисеев С.Г., Остаточников В.А., Семениов Д.И. // Квант. электрон. 2012. Т. 42. С. 557.

[17] Spanier J.E., Herman I.P. // Phys. Rev. B. 2000. V. 61. P. 10437.

[18] Drachev P., Buin A.K., Nakotte H., Shalaev V.M. // Nano Lett. 2004. V. 4. N 8. P. 1535-1539.

[19] Govyadinov A.A., Panasyuk G.Y., Schotland J.C., Markel V.A. // Phys. Rev. B. 2011. V. 84. N 15. P. 155461-1-12.

[20] Maxwell-Garnett J.C. // Philos. Trans. R. Soc. London. A. 1904. V. 203. P. 385.

[21] Головань Л.А., Тимошенко В.Ю., Кашкаров П.К. // УФН. 2007. T. 177. № 6. С. 619-638.

[22] Елисеева С.В., Наседкина Ю.Ф., Семенцов Д.И. // Опт. и спектр. 2014. Т. 117. № 6. C. 50-58.

[23] Noskov R.E., Smirnova D.A., Kivshar Yu.S. // Opt. Lett. 2013. V. 38. C. 2554-2556.

[24] Zhang X.J., Ji W., Tang S.H. // JOSA. B. 1997. V. 14. P. 1951.

[25] Степанов А.Л. // ЖТФ. 2004. Т. 74. С. 1-12.

[26] Ganeev R.A., Ryasnyansky A.I., Stepanov A.L., Usmanov D.T. // Opt. Quant. Electron. 2004. V. 36. P. 949-960.

[27] Борн М., Воль $\oint$ Э. Основы оптики. М.: Наука, 1973. 